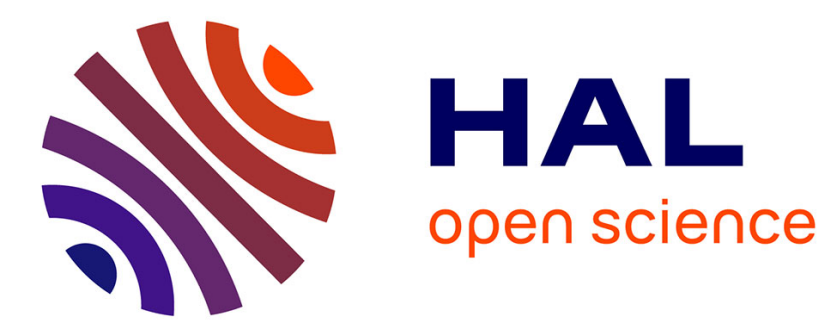

\title{
Link State Routing in Wireless Ad-Hoc Networks
}

Cédric Adjih, Emmanuel Baccelli, Philippe Jacquet

\section{To cite this version:}

Cédric Adjih, Emmanuel Baccelli, Philippe Jacquet. Link State Routing in Wireless Ad-Hoc Networks. MILCOM'03 - The IEEE Military Communications Conference, Oct 2003, Boston, United States. pp.1274 - 1279, 10.1109/MILCOM.2003.1290410 . hal-00781832

\section{HAL Id: hal-00781832 \\ https://hal.inria.fr/hal-00781832}

Submitted on 28 Jan 2013

HAL is a multi-disciplinary open access archive for the deposit and dissemination of scientific research documents, whether they are published or not. The documents may come from teaching and research institutions in France or abroad, or from public or private research centers.
L'archive ouverte pluridisciplinaire HAL, est destinée au dépôt et à la diffusion de documents scientifiques de niveau recherche, publiés ou non, émanant des établissements d'enseignement et de recherche français ou étrangers, des laboratoires publics ou privés. 


\title{
LINK STATE ROUTING IN WIRELESS AD-HOC NETWORKS
}

\author{
C. Adjih, E. Baccelli, P. Jacquet \\ Hipercom Project, INRIA Rocquencourt BP 105, 78153 Le Chesnay Cedex, FRANCE \\ \{Cedric.Adjih, Emmanuel.Baccelli, Philippe.Jacquet\}@inria.fr
}

\begin{abstract}
Link state has been the dominating IGP routing technology in IP networks for more than a decade. With the advent of wireless ad-hoc networking, the need for light, efficient and robust routing makes it a good candidate to be also used in this constrained environment. One of the key issue in ad-hoc networking is the lack of bandwidth. Thus, in this paper we examine the overhead due to link state routing in an ad-hoc network. More precisely we study the classic link-state protocol OSPF and OLSR, a link state protocol that was developed specifically for ad-hoc networks. Further, we study how this overhead evolves when the node density increases. The last part of the paper presents directions towards extending OSPF for wireless and ad-hoc networks, which would make it a favorite to seamlessly unify wired and mobile IP networks.
\end{abstract}

\section{INTRODUCTION}

Since the beginning of the 1990s, essentially with OSPF (Open Shortest Path First, see [3]) and IS-IS (Intermediate System to Intermediate System, see [6]), link state routing has taken over as the dominating technology for Interior Gateway routing on IP networks. These protocols have succeeded to others like RIP (Routing Information Protocol, see [7]), that were based on a different routing technology, namely distance vector. This technology was rather simple but however plagued with routing loops and slow convergence, not to mention divergence with the famous "count to infinity" behavior. Link state routing has been introduced purposely to radically improve on those issues.

The approach taken by link state protocols is that of a distributed database which is replicated throughout the routing domain, as opposed to the distributed computation with distance vector. More precisely each node starts by describing its local environment i.e. the state of the links with its neighbors - neighbor sensing is done via the periodic sending/receiving of simple "Hello" packets. These descriptions are then flooded to all the other routing nodes, contributing pieces to a database which therefore first contains the descriptions of all the nodes, and second is present in each node.

This link state database is kept up-to-date in all nodes by the same flooding mechanism, and such periodically as well as occasionally in case of change in some node's neighborhood. Each node then possesses enough information at any time to build a view of the entire network and to compute the shortest paths to any other node (with the help of a Djikstra-like algorithm).

At the price of having considerably more complex mechanisms than with distance vector, the link state approach indeed succeeds in producing protocols that don't diverge, and, even better, that converge faster while avoiding routing loops. The canonical example for such a protocol is OSPF (see [3] [9]), which second version, namely OSPFv2, has been very widely deployed and used over the last decade.

\section{Ad-Hoc Networking and Link State Routing}

Since a few years now, the hottest trend in networking is the use of wireless links. Examples are numerous, from cordless phones to GSM and from Bluetooth to $\mathrm{Wi}-\mathrm{Fi}$, everything seems to go towards a wireless world. Going further on this path, ad-hoc networking is perhaps the most hardcore example of this trend: a manet (manet for Mobile Ad-hoc NETwork, see [8]) is essentially a network of equal mobile nodes communicating via multi-hop wireless links, and such with a dynamical, self-organized behavior - namely without central control. The most immediate example of possible application is a fleet of mobile military devices on a battlefield. But with these specificities, ad-hoc networks would indeed open a new era, as far as networking and automation is concerned their applications would no doubt end up to be countless in the future.

However, if usual wired IP networks have been unanimously tamed with protocols like OSPF, it is not yet the case for ad-hoc networks. When compared to their wired counter-part, manets are indeed a much harsher environment: they feature a higher topology change rate, a lower bandwidth, a lower transmission quality, a lower security, as well as more scaling problems and possibly some energy and memory constraints on the mobile nodes. These problems are mainly inherent to mobility and to the use of radio links.

Lately, ad-hoc networking has been subject to a lot of work, and numerous specific protocols have been developed. One of these protocols is OLSR (Optimized Link State Routing, see [4]), which is a link state protocol essentially very close the archetype OSPF. Indeed, after a decade of experience on wired networks, the proven qualities of link state technology (robustness, quick convergence, and relative lightness) make it a very good candidate to be also used in an ad-hoc environment. OLSR works well (see [1]), and is considered one of the most prominent ad-hoc networking solution.

The question is: if OSPF and OSLR are so similar, why is OSPF not again simply used as is on manets? There are in fact several issues with the use of OSPF in ad-hoc networks. But then why bother doing this if other protocols (like OSLR) work fine on manets? The truth is that having OSPF run also on ad-hoc networks is a very seducing proposal for several reasons. First because of 
OSPF's legacy: it is extremely well deployed, known, and renowned, therefore possibly facilitating greatly the deployment of ad-hoc networking if it could be used for that. Second, it would allow to seamlessly unify wired and wireless IP networking under a unique routing solution. This is very interesting industry-wise in terms of maintenance and costs. On the other hand this is also interesting for users in terms of abstraction and ease of use.

The last section of this paper tackles the different problems with OSPF on ad-hoc networks and extracts directions towards adapting this protocol for this specific environment. But first we will focus on the overhead issue: as mentionned earlier, a fundamental constraint in manets is the lack of bandwidth as compared to wired networks. Therefore the amount of overhead due to the routing is key to the performance of a protocol used on manets. The next sections will analyze via mathematical modeling the overhead due to link state routing in ad-hoc networks with OSPF on one hand and with OLSR on the other hand.

\section{AD-HOC NETWORK MODELING}

In a network running a link state routing protocol, the number of hops between any random source and destination increases when the neighborhood size decreases, this way augmenting the overhead due to control traffic. Following this path, we will study the control overhead via the analysis of the maximum manageable neighborhood size.

In an awarded paper, K. Gupta et al. have shown via information theory that when the size of the network $N$ increases, the neighborhood size is $O(\log N)$ and its diameter in number of hops increases at least in $\sqrt{N} / \log N$. In this paper, we will show via a simple model and with existing routing protocols that the neighbor size tends to be constant and the number of hops increases in $\sqrt{N}$ when $N$ increases. The constant in front of this order of magnitude depends on the nature of the routing protocol and can vary greatly. We analyze the impact of the routing protocol on this constant: we show that the constant greatly varies between the pure link state protocol (OSPF) and the optimized link state protocol (OLSR). In particular we show that when the network size increases the maximum manageable neighbor size is respectively 12 for OSPF and 50 for OLSR.

\section{Propagation Model}

We consider the following model: time is slotted and all the mobiles are synchronized with transmissions on beginning of slots. We consider an area of arbitrary size $\mathcal{A}$ (we will ignore border effects). $N$ transmitters are distributed according to a Poisson process. We call $\lambda$ the density of transmitters per slot and per square area unit. We have $\lambda=f N / \mathcal{A}$ where $f$ is the rate of packets transmission per slot and per node.

Let $\mathrm{X}$ be a node at a random position (we ignore border effects). We assume that all nodes transmit at the same nominal power. The reception signal at distance $r$ is $P(r)=r^{-\alpha}$ with $\alpha>2$. Typically $\alpha=2.5$. Let $W$ be the signal intensity received by node $\mathrm{X}$ at a random slot. Quantity $W$ is a random variable: let $w(x)$ be its density function. In [5] it is shown that the Laplace transform of $w(x), \tilde{w}(\theta)=\int w(x) e^{-x \theta} d x$ satisfies the identity:

$$
\tilde{w}(\theta)=\exp \left(2 \pi \lambda \int_{0}^{\infty}\left(e^{-\theta r^{-\alpha}}-1\right) r d r\right) .
$$

Using standard algebra we get

$$
\tilde{w}(\theta)=\exp \left(-\lambda \pi \Gamma\left(1-\frac{2}{\alpha}\right) \theta^{2 / \alpha}\right) .
$$

If the node location map was a line instead of an area (consider a sequence of mobiles nodes on a road) then we would have

$$
\tilde{w}(\theta)=\exp \left(-\lambda \Gamma\left(1-\frac{1}{\alpha}\right) \theta^{1 / \alpha}\right) .
$$

If, instead the location map was a volume (consider aircrafts network), then

$$
\tilde{w}(\theta)=\exp \left(-\frac{4}{3} \lambda \pi \Gamma\left(1-\frac{3}{\alpha}\right) \theta^{3 / \alpha}\right) .
$$

In the following we restrict ourselves on a 2D map.

\section{Neighbor Model}

A node is considered neighbor of another node if the probability of receiving hellos from each other is greater than a certain threshold $p_{0}$. Typically $p_{0}=1 / 3$. We assume that a packet can be decoded if its signal-over-noise is greater than a given threshold $K$. Typically $K=10$. Therefore another node is neighbor if its distance $r$ is such that $P\left(W<r^{-\alpha} / K\right)>p_{0}$, i.e. when $r<r(\lambda)$ where $r(\lambda)$ is the critical radius such that $\int_{0}^{r(\lambda)} w(x) d x=p_{0}$.

By simple algebra it comes that $r(\lambda)=\lambda^{-1 / 2} r(1)$, and the surface covered by the radius $r(\lambda)$ is the neighborhood area $\sigma(\lambda)=\frac{\sigma(1)}{\lambda}$.

\section{Computation of $\sigma(1)$}

In order to simplify we set $C=\pi \Gamma\left(1-\frac{2}{\alpha}\right)$ and $\gamma=\frac{2}{\alpha}$. By application of reverse Laplace we have:

$$
P(W<x)=\frac{1}{2 i \pi} \int_{-i \infty}^{+i \infty} \frac{\tilde{w}(\theta)}{\theta} e^{\theta x} d \theta .
$$

Expanding $\tilde{w}(\theta)=\sum_{n} \frac{(-C)^{n}}{n !} \theta^{n \gamma}$, we get

$$
P(W<x)=\frac{1}{2 i \pi} \sum_{n} \frac{(-C)^{n}}{n !} \int_{-i \infty}^{+i \infty} \theta^{n \gamma-1} e^{\theta x} d \theta
$$

By bending the integration path toward the negative axis we get

$$
\begin{aligned}
\frac{1}{2 i \pi} \int_{-i \infty}^{+i \infty} \theta^{n \gamma-1} e^{\theta x} d \theta & =\frac{\sin (\pi n \gamma)}{\pi} \int_{0}^{\infty} \theta^{n \gamma-1} e^{-\theta x} d \theta \\
& =\frac{\sin (\pi n \gamma)}{\pi} \Gamma(n \gamma) x^{-n \gamma}
\end{aligned}
$$

Figure 1 shows the plot of $P(W<x)$ versus $x$ for $\alpha=2.5$ and $\lambda=$ 1. Notice that $P(W<x)$ reaches $p_{0}=1 / 3$ close to $x=x_{0}=20$. Therefore $r(1)=\left(x_{0} K\right)^{-1 / \alpha} \approx 0.12$. Therefore $\sigma(1) \approx 0.045$. 


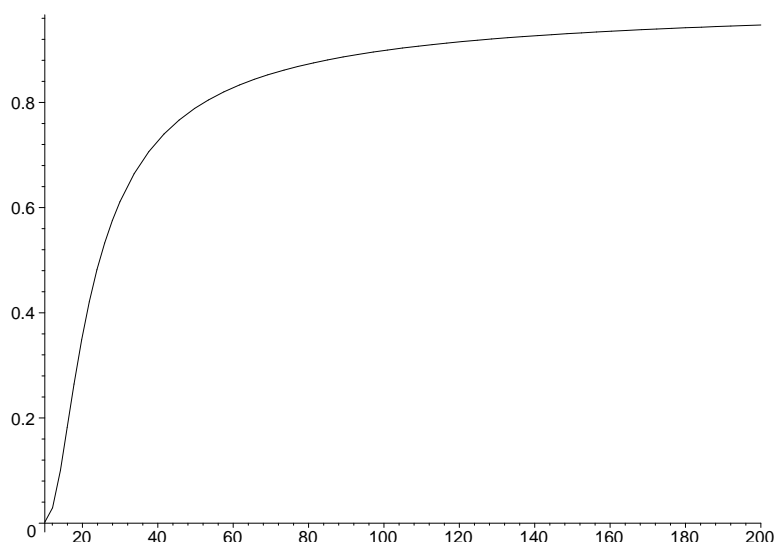

Figure 1: Quantity $P(W<x)$ versus $x$ for $\alpha=2.5$, no fading.

\section{Fading Model}

The propagation of waves in presence of random obstacles experiences a random fading. Fading is usually modelled with the introduction of a random factor $F$ to signal attenuation at distance $r: r^{-\alpha}$. For example $\log F$ is uniform on $[-v, v]$. In this case we have a new expression of $\tilde{w}(\theta)$ :

$$
\tilde{w}(\theta)=\exp \left(-\pi \lambda \Gamma\left(1-\frac{2}{\alpha}\right) \phi\left(-\frac{2}{\alpha}\right) \theta^{2 / \alpha}\right)
$$

with $\phi(s)=E\left(F^{-s}\right)$, the Dirichlet transform of the fading. When fading is uniform on $[-v, v]$ we have $\phi(s)=\frac{\sinh (s v)}{s v}$. For any given real number $x$ we also have $P(W<x F)$ equalling

$$
\sum_{n} \frac{(-C F(-\gamma))^{n} \sin (\pi n \gamma)}{\pi n !} \Gamma(n \gamma) \phi(n \gamma) x^{-n \gamma}
$$

which helps the computation of $\sigma(1)$ with fading.

\section{Network Topology}

We consider that the network is uniformly distributed with an uniform density $\nu$ on an area of size $\mathcal{A}$. The total number of nodes in the network is $N=\nu \mathcal{A}$. If $\lambda$ is the traffic density in the network, then the average number of neighbors per node is $M=\sigma(\lambda) \nu=\sigma(1) \frac{\nu}{\lambda}$.

\section{LINK STATE OVERHEAD}

We consider the following two link state protocols: OSPF [3] and OLSR [4]. The aim is to derive the traffic density generated by the control packets of the protocols. There are two sources of control traffic: neighbor sensing on one hand and topology discovery on the other hand.

Neighbor sensing is the same for all link state protocols: it consists in each node periodically transmitting a hello message containing the list of neighbors heard by the node. By comparing their lists the nodes can determine the set of neighbors with which they have symmetric links.

Let $h$ be the rate at which nodes refresh their neighbor information base and let $B$ be the maximum number of node identifiers that a slot can contain. For a network with the capacity of WiFi (1-10 Mbps) we have $B=100$ and 1,000 slots per second. An OLSR node generates hellos every $2 \mathrm{sec}$, i.e. $h=1 / 2000$. If the neighbor list exceeds $B$ then the node generates several hellos per update period and distributes the neighbor list among these several hellos. The node must generate $\left\lceil\frac{M}{B}\right\rceil$ hellos per hello period. Therefore the hellos lead to a traffic density of $h \nu\left\lceil\frac{M}{B}\right\rceil$. Omitting fractional part we get

$$
\lambda=h \nu \frac{M}{B}
$$

if the hellos is the only source of control traffic. Since $M=\sigma(1) \frac{\nu}{\lambda}$ we get

$$
\frac{\sigma(1)}{M}=h \frac{M}{B} .
$$

In fact this only an upper bound because the network size might be smaller than $\sigma(1)$. Therefore the maximum manageable neighbor size with only the hello control traffic is $\sqrt{B \sigma(1) / h} \approx 95$

Topology discovery varies between protocols. With OSPF, each node periodically broadcasts its list of adjacent links in an LSA (Link State Advertisement) message, the nodes re-broadcast the LSA towards their neighbors. In OLSR the nodes periodically broadcast TC (Topology Control) messages containing only a subset of their adjacent links - the MPR (MultiPoint Relay) selector links. Moreover, only a subset of the neighbors (the MPR nodes) re-broadcast the TC messages. However we will assume that in both protocols the topology discovery update period is the same, in order to compare two protocols with the same agility to adapt their topology to mobility. For instance, OLSR's TC rate per node is $\tau=1 / 5000$.

\section{Model for OSPF}

The idea is to express $\lambda$ only in function of the protocol overhead. We consider no other traffic than the signalling protocol. Therefore the performance will be best case. In OSPF a node periodically

1. transmits Hellos with rate $h$. A Hello contains the list of all neighbor identifiers (if the list is too long, it will take several packets on several slots);

2. transmits LSAs with rate $\tau$. An LSA contains the list of all adjacent links;

3. retransmits received LSAs with large jitter, to all neighbors separately (one copy per neighbor).

Therefore the traffic density satisfies the following identity:

$$
\lambda=h\left\lceil\frac{M}{B}\right\rceil+\tau \nu N M\left\lceil\frac{M}{B}\right\rceil .
$$

For the following we drop the ceil factor:

$$
\lambda=h \nu \frac{M}{B}+\tau \nu N \frac{M^{2}}{B} .
$$

Using $M=\sigma(1) \frac{\nu}{\lambda}$ we have the identity

$$
\frac{\sigma(1) B}{M}=h M+\tau N M^{2} .
$$


This allows to put in relation the total size of the network $N$ with the average neighborhood size $M$. When $N$ increases, $M$ decreases. The minimum size for $M$ is one, below which the network does not have a significant connected component. In fact if one would have a full connected network, the threshold should be raised to $\log N$. The limit $M=1$ puts the maximum size the network to $N_{\max }$

$$
N_{\max }=(\sigma(1) B-h) \frac{1}{\tau} .
$$

We have $N_{\max }=25,000$. When the network size decreases, it reaches a level where $N=M$. Below this level the network is only one hop, the control traffic cannot saturate the neighborhood. The mono-hop level for OSPF from (13) is $N=12$.

\section{OSPF modified: $O S P F-B$}

We propose an adaptation of OSPF which tries to reduce the overhead. In OSPF-B the nodes broadcast the LSA only once, instead of duplicated in several copies to each neighbor.

In this case the equation (13) should be rewritten in

$$
\frac{\sigma(1) B}{M}=h M+\tau N M
$$

The mono-hop level for OSPF-B is $N=25$.

\section{Model for OLSR}

In OLSR a node periodically

1. transmits Hellos with rate $h$. A Hello contains the list of all neighbor identifiers (if the list is too long, it will take several packets on several slots);

2. transmits TCs with rate $\tau$. A TC contains the list of neighbors having selected the node as MPR (MPR selectors);

3. retransmits received TCs only once and with large jitter, and this only when the node is MPR of the first reception.

Let $M_{r}$ be the average number of MPR of a node with neighbor size $M$. Since the model of network is the disk unit graph it turns from [1] that $M_{r} \leq\left(9 \pi^{2} M\right)^{1 / 3}$. Simulations show that $M_{r} \sim \beta M^{1 / 3}$ when $M \rightarrow \infty$ with $\beta \approx 5$ (see figure fig-mpr). Simulations were performed up to $M=6,000,000$.

In [2] we prove that the MPR flooding costs $M_{r} N / M$ retransmissions in average. Therefore we get the traffic density identity:

$$
\frac{\sigma(1) B}{M}=h M+\tau\left(M_{r}\right)^{2} \frac{N}{M} .
$$

The mono-hop level of OLSR is $N=50$.

\section{OLSR modified: F-OLSR}

In this section we introduce F-OLSR, for Full Optimized Link State Routing, a version of OLSR less optimized in terms of overhead,

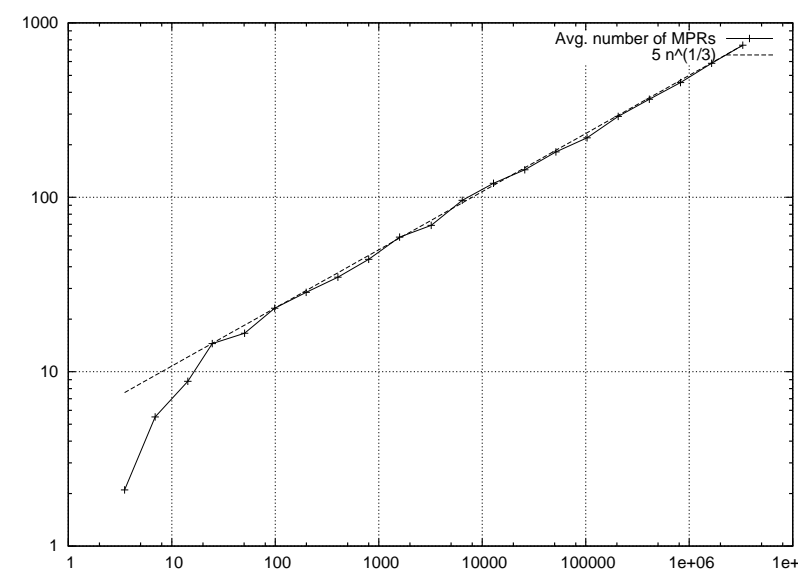

Figure 2: Average MPR set of a node versus neighborhood size.

which reports the topology completely (instead of partially). In FOLSR the TCs contain the list of all the adjacent links. Therefore every node has the knowledge of the complete link state of the network instead of a knowledge restricted to MPR links. The TCs are still forwarded via MPR nodes. The equation for F-OLSR is:

$$
\frac{\sigma(1) B}{M}=h M+\tau M_{r} N
$$

and the mono-hop level is at $N=34$.

On figure 3 we show the respective neighborhood size versus network size $N$ for the two versions of OSPF. On figure 4 we show the respective neighborhood size versus network size for the two versions of OLSR. Figure 5 compares OSPF and OLSR on the same plot. If we take as hop number estimate the square root of the ratio network size over neighborhood size, then we have figure 6.

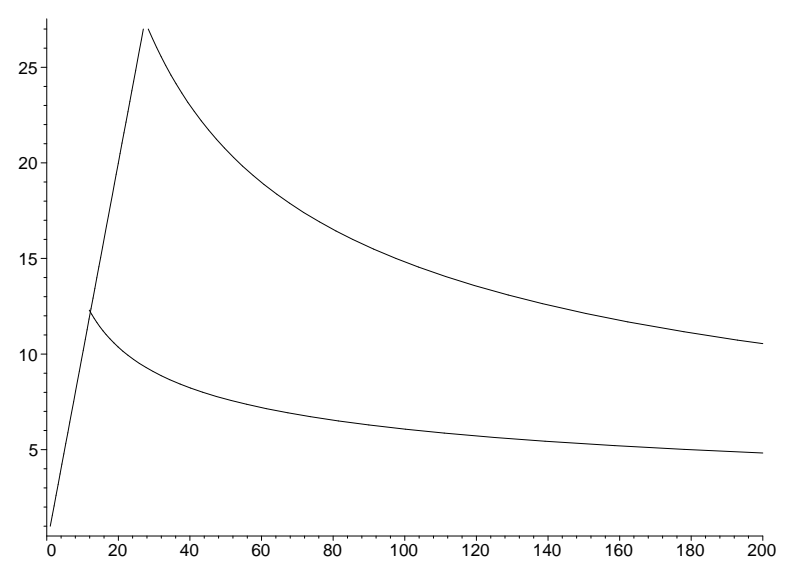

Figure 3: Neighborhood size versus the network size, $\alpha=2.5$, no fading, respectively for OSPF (bottom) and OSPF-B (top).

\section{Conclusion on Modelling}

We have compared the performance of four different link state routing algorithms with a simple interference model. We basically show 


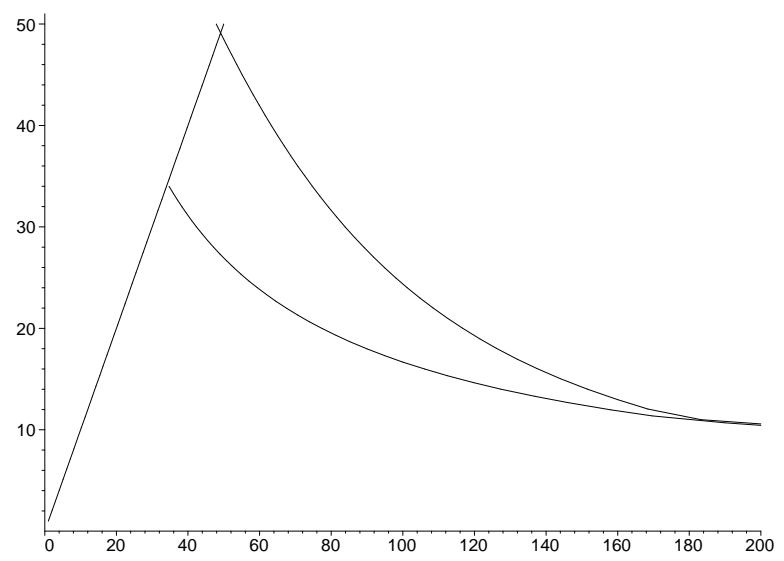

Figure 4: Neighborhood size versus the network size, $\alpha=2.5$, no fading, respectively for F-OLSR (bottom) and OLSR (top).

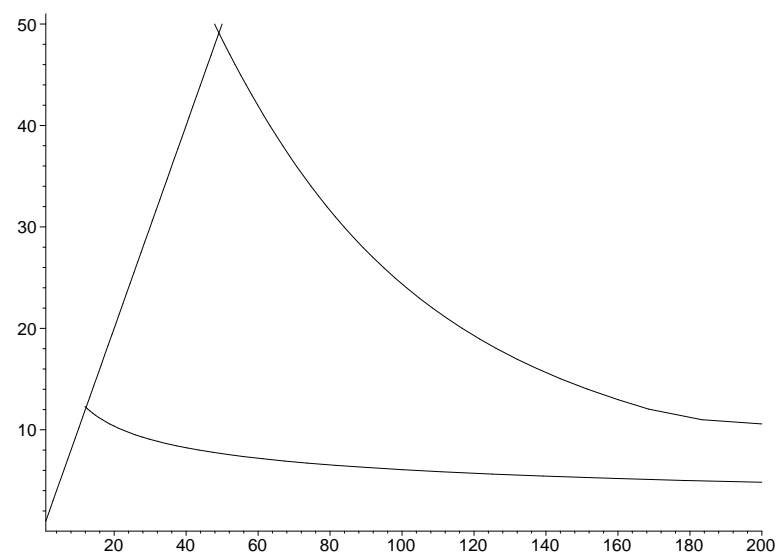

Figure 5: Neighborhood size versus the network size, $\alpha=2.5$, no fading, respectively for OSPF (bottom) and OLSR (top).

that the use of a specific routing algorithm impacts the maximum manageable neighborhood size, which can vary greatly from one protocol to another - when this size decreases, the overhead due to the routing protocol increases. With a 2D model without fading, and with attenuation coefficient $\alpha=2.5$, the maximum neighborhood size is limited to 12 with OSPF, whereas it can rise up to 50 with OLSR - the same update rate parameters are used for both protocols. With this respect, OLSR clearly outperforms OSPF in an ad-hoc environment, and performs well overall, knowing that this size is anyway limited to 95 due to neighbor sensing control traffic.

It is important to note that this analysis is based on the assumptions that time is slotted and that mobile nodes are synchronized. This is namely not the case with CSMA and 802.11, which are used in practice. Experience with those shows that while OLSR offers comparable results, OSPF performs significantly worse than in our slotted model, and such to the point that OSPF control traffic on its own rapidly overflows CSMA. The protocol is then of course unable to converge - even basic neighbor sensing is hampered, with most Hello messages being unable to go through.

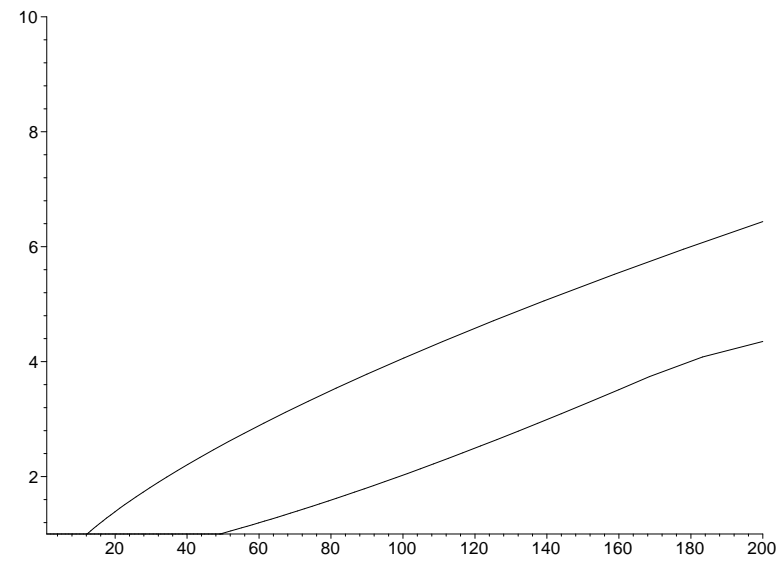

Figure 6: Hop number estimated diameter of the network versus network size, $\alpha=2.5$, no fading, respectively for OLSR (bottom) and OSPF (top).

\section{TOWARDS ADAPTING OSPF FOR AD-HOC NETWORKS}

As we have already suggested, it is a very seducing idea to run OSPF on manets also. OLSR and OSPF are very close in essence and hence, it is indeed natural to think about this being quite possible. Nevertheless, there are numerous problems with OSPF on mobile wireless networks. We will summarize them now, after which we will give possible general directions towards adapting OSPF for these networks.

\section{Issues with OSPF on MANETS}

We have seen in the previous sections that OSPF's overhead is a big handicap in an ad-hoc environment, and makes it perform quite poorly compared to OLSR in most cases. More precisely, the prominent issues with OSPF are the following three.

First, OSPF features a mechanism aiming at optimizing the retransmissions on interfaces featuring broadcast capabilities: the Designated Router (DR) mechanism. Optimizing retransmissions is crucial in an ad-hoc network, both in terms of overhead and in terms of radio collisions, and not only is the DR approach not efficient in an ad-hoc environment, but OSPF even fails to converge because the mechanism doesn't work in face of the "hidden node" problem (i.e. a common wireless configuration where some node hears a node that other nodes don't).

Second, OSPF uses unicasted acknowledgements, in order to make the flooding of LSAs more reliable. In an ad-hoc environment, ACKs are very likely to get synchronized and to collide in series on the radio link, potentially leading the network to a break down. In any case, this is serious threat to functionality and performance.

And third, OSPF features hierarchical routing, allowing to segregate nodes in distinct ensembles called OSPF areas. Area mobility (i.e. when a mobile node moves from an OSPF area to another) is an issue in terms of dynamic configuration, security, authentication and prefix routing. Note that nodes running OSPFv2 with different 
parameters (i.e. IP prefix, area ID, security parameters...) will essentially refuse to become neighbors even if they can "hear" each other. A new version of OSPF (i.e. OSPFv3) works around some of these problems, but it is not mature yet.

Aside of these three main issues, additional tuning should also be considered for several other aspects such as, for instance, the metric on wireless links especially as compared to wired links, the use of the Database Exchange mechanism (a mechanism aiming at jump-starting a new neighbor relationship by exchange of link state databases), the amount of LSAs on the wireless parts and their life time, or the flooding mechanism.

\section{Directions in View to Extend OSPF for MANETs}

After this, the first thing that comes to mind is the need for a new extension. Just like a specific OSPF interface was designed for different technologies like Ethernet (broadcast interface) or for Frame Relay (Point-to-Multipoint interface), a new OSPF interface specially designed for wireless networks is needed, taking into account their specificities in its neighbor discovery and maintenance, its database synchronization and its abstraction mechanisms (see [10]). More generally, the goal is then to define an extension of OSPF that adapts well to ad-hoc specificities, while OSPF runs unaltered on usual networks and interfaces. This is a must have in order to be backward compatible with wired networks running usual OSPF and to achieve the seamless unity of wired and wireless IP networking.

As we have seen in the previous sections, the MPR mechanism is a very good candidate to replace the DR concept on these radio interfaces which have native broadcast capabilities. MPRs are far more suitable in this environment as they were designed for it contrary to the DR mechanism. Note that to a certain extent, the Designated Router election can be seen as a special case of MPR selection.

The use of implicit acknowledgement on the wireless interface should be as extensive as possible, since it is naturally there: packets are retransmitted also on the same radio interface they were received on - note that this is a slight difference with wired OSPF. Therefore a source should be able to take a re-transmitted packet as implicit acknowledgement. More generally and for instance, OLSR works with the inherent unreliability of the radio medium by not requiring reliable transmission of control messages, but just relies on them being periodically sent in order to sustain an occasional loss. The main concern about this is that link state databases might not be synchronized as well as they are with usual OSPF. However it still works, and if such a scheme is used anyway, note that in our case only mobile nodes will face this slight concern, while any other node that has a usual OSPF interface will be as synchronized as ever. If the use of explicit acknowledgements on wireless interfaces is required, further work has to be completed in order to design an optimized scheme that would fit an ad-hoc environment.

The problems due to area mobility can be separated from the others: they are not about basic routing, but rather about hierarchical routing. They can therefore be solved independently. We will not discuss them further in this paper, but the use of OSPFv3 along with a dynamical authentication/configuration mechanism kept as simple as possible might be a good way to solve them (see [10]).

\section{CONCLUSIONS \& FUTURE WORK}

In this paper we have analyzed link state algorithms for ad-hoc routing with the comparison on several aspects between the archetype link state protocol OSPF and the ad-hoc routing protocol OSLR. And this more specifically in terms of overhead, which is the main constraint in an ad-hoc environment - along with fast topology change rate. We have shown that OSPF performs quite poorly in most cases, especially as compared to OLSR - this is consistent with simulations carried out independently from our work. Given that having OSPF perform also on ad-hoc networks would simply unify wired and wireless IP networking (which would make this solution a market favorite) we have extracted several general directions that can be envisioned towards adapting OSPF for ad-hoc networking, with a new extension to be fully designed.

\section{REFERENCES}

[1] P. Jacquet, A. Laouiti, P. Minet, L. Viennot, "Performance evaluation of multipoint relaying in mobile ad hoc networks," Networking 2002, Pisa, 2002.

[2] C. Adjih, P. Jacquet, L. Viennot, "Computing connected dominating set with multipoint relays,'INRIA Research Report RR-4597, 2002.

[3] J. Moy, "OSPF version 2," RFC 2328, http://ietf.org/rfc/rfc2328.txt, 1998.

[4] T. Clausen, P. Jacquet, A. Laouiti, P. Minet, P. Muhlethaler, A. Qayyum, L. Viennot, "Optimized Link State Routing Protocol," draft MANET-IETF, http://www.ietf.org/internet-drafts/draft-ietfmanet-olsr-07.txt, 2002.

[5] P. Jacquet, "Elément de théorie analytique de l'information, modélisation et évaluation de performances," INRIA Research Report RR-3505, http://www.inria.fr/rrrt/rr-3505.html, 1998.

[6] D. Oran, “OSI IS-IS Intra-domain Routing Protocol," RFC 1142, http://ietf.org/rfc/rfc1142.txt, 1990.

[7] G. Malkin, “RIP Version 2,” RFC 2453, http://ietf.org/rfc/rfc2453.txt, 1998.

[8] S. Corson, J. Macker, "Mobile Ad hoc Networking (MANET): Routing Protocol Performance Issues and Evaluation Considerations," RFC 2501, http://ietf.org/rfc/rfc2501.txt, 1999.

[9] J. Moy, "OSPF, Anatomy of an Internet Routing Protocol," AddisonWesley, 1998.

[10] F. Baker, "An outsider's view of MANET," Internet-Draft: draft-bakermanet-review-01.txt (expired), March 2002. 